\title{
Correlation Studies on Ancient Irrigation Tanks in Sri Lanka
}

\section{O. A. Gunawardena and R. P. de Silva}

Abstract: Arumugam (1957) outlined a simple method of determining the spill dimensions of a village irrigation tank. A study was carried out to see if the ancient tank builders have followed this method and the very high correlation coefficients $(>0.91)$ suggest that these builders may have known the method proposed by Arumugam. Of course the study could be carried out only in the case of 8 tanks where the relevant data are available. The correlations are not that strong when these tanks were restored as now modern methods of designing a spill is available.

Keywords: Irrigation tanks, spill design, ancient Sri Lanka

\section{Introduction}

Sri Lanka (then Ceylon) is famousfor its ancient irrigation systems consisting of many tanks (about 25,000 of them), canals and also for inventions of our own such as the Bisokotuwa (400 -300 BC) or valve pit, which enabled a controlled off take of water from tanks. Figure 1 below shows the distribution of some of these ancient tanks in Sri Lanka.
Out of these 25,000 or so tanks (referred to as Weva and Kulum in some instances), many are village tanks, built using a technology known perhaps only to the ancient builders. This paper describes a study where an investigation was carried out to see if the ancient tank builders also have followed the method given by Arumugam (1957) forvillage tank spill analysis leading to the design of the spill

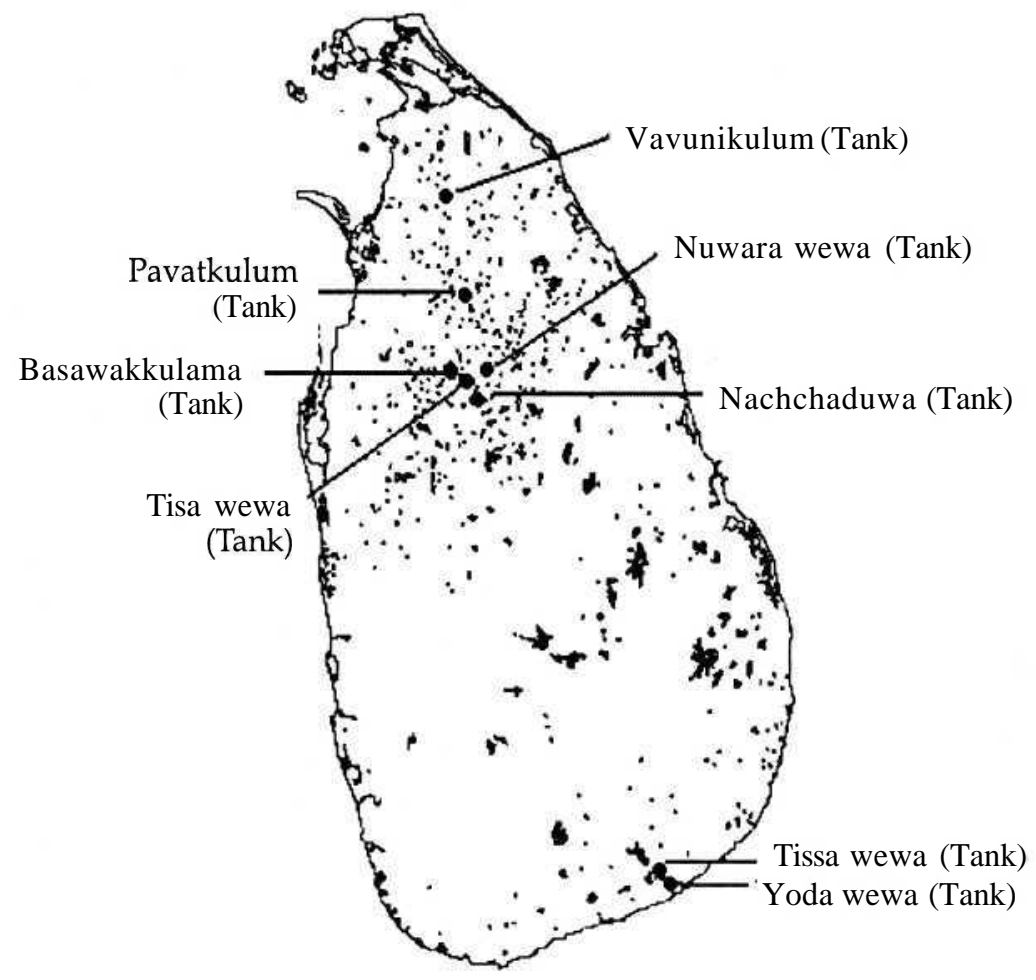

Fig. 1 - Location map of some of the ancient tanks in Sri Lanka (Tanks considered in this study are identified) 
The method by Arumugam (1957), one of the simplest of methods of a village tank spill analysis, is to assume that the maximum flood inflow is obtained by Dickens' formula at a constant rate for 12 hours and to equate this flood to the tank detention and spillage, whereby the required spill design is obtained. The method is illustrated graphically in Fig. 2.

\section{Materials and Methods}

The relevant data (such as catchment area of a tank, area of tank at full supply level, length of original bund, width of bund top, full supply level (FSL), bund top level (BTL), length of spill etc) were obtained from various sources (Parker, 1909; Brohier, 1934; Arumugam, 1969 and Dharmasena, 1988).

\section{TYPE SPILL ANALYSIS DIAGRAM}

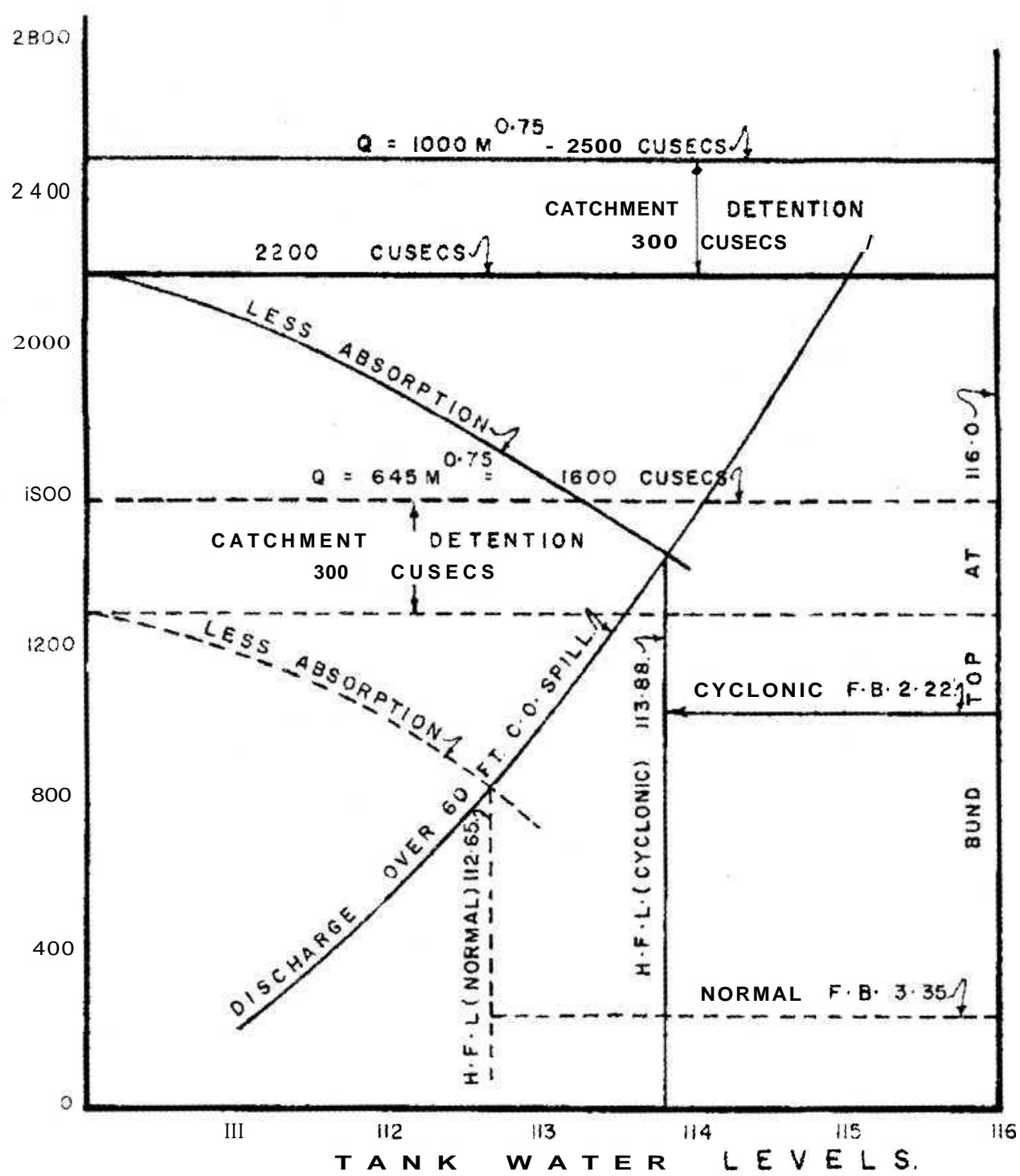

Fig. 2 - Graphical illustration of Arumugam's Spill Analysis Method (Arumugam, 1957). 
Dharmasena (1988) has published an article on "SomeAspect of Ancient Irrigation Works in Sri Lankd" in the Journal "Engineer", September December 1988. From H Parker's data, he prepared the parameters of 11 tanks. These parameters are given in Table 1 (top row foreach tank) along with the present day parameters (bottom row). Only 8 of the tanks have the ancient spill parameters given.

To estimate the inflow flood, Dickens' formula $Q_{d}$ $=\mathrm{CM}^{3 / 4}$ where $\mathrm{Q}_{\mathrm{d}}$ is the flood inflow into the tank in cusecs was used. Here, $\mathrm{C}$ is a coefficient taken by us as 800 (for a discussion on the selection of
C, please refer Arumugam, 1957) and $M$ is the catchment area of the tank in square miles. This flood inflow was correlated to $\left(\mathrm{Q}=3.3 \mathrm{LH}^{3 / 2}\right)+$ $\mathrm{AH}$ where $\mathrm{Q}$ is the flood discharge over the spill, $\mathrm{L}$ is the spill length, $\mathrm{H}$ is the head over the spill and $A$ is the water spread area. Here $Q$ is in cusecs, $\mathrm{L}$ and $\mathrm{H}$ are in feet and $\mathrm{A}$ is in acres. The detention term $\mathrm{AH}$ is a volume in Acft. but over 12 hours can be considered as cusecs, because 1 cusec for 12 hours is 1 Acft. and the high flood level is attained at 12 hours after the commencement of the flood inflow.

The water spread areas were obtained from Parker's Ancient Ceylon (1909).

Table 1 Comparison of Tank parameters for ancient and restored tanks

(Source: Dharmasena, 1988; Arumugam, 1969)

\begin{tabular}{|c|c|c|c|c|c|c|c|c|c|}
\hline & $\begin{array}{c}\text { Name of tank \& } \\
\text { approximate } \\
\text { construction date }\end{array}$ & $\begin{array}{c}\text { Catch } \\
\text {-ment } \\
\text { area } \\
\text { sq.mls. }\end{array}$ & $\begin{array}{l}\text { Capacity } \\
\text { (Ac.ft.) }\end{array}$ & $\begin{array}{l}\text { Original } \\
\text { bund } \\
\text { length in } \\
\text { ft. }\end{array}$ & $\begin{array}{c}\text { Top width } \\
\text { in } \mathrm{ft} .\end{array}$ & $\begin{array}{c}\text { Full } \\
\text { supply } \\
\text { level - } \\
\text { FSL (ft) }\end{array}$ & $\begin{array}{l}\text { Bund top } \\
\text { level - } \\
\text { BTL (ft) }\end{array}$ & $\begin{array}{l}\text { Length of } \\
\text { spill } \\
\text { in } \mathrm{ft} .\end{array}$ & $\begin{array}{c}\mathrm{U} / \mathrm{S} \\
\& \mathrm{D} / \mathrm{S} / \\
\text { slopes }\end{array}$ \\
\hline 1 & $\begin{array}{c}\text { Panda Wewa . } \\
400 \text { B.C }\end{array}$ & $\begin{array}{l}\text { NA } \\
\text { NA }\end{array}$ & 2400 & $\begin{array}{l}8400 \\
\text { NA }\end{array}$ & $\begin{array}{r}8.0 \\
\text { NA }\end{array}$ & $\begin{array}{l}13.0 \\
\text { NA }\end{array}$ & $\begin{array}{l}22.0 \\
\text { NA }\end{array}$ & $\begin{array}{l}250 \\
\text { NA }\end{array}$ & $\begin{array}{c}2.5 / 2.5 \\
\text { NA }\end{array}$ \\
\hline 2 & $\begin{array}{c}\text { Basawakkulama } \\
\text { Tank } \\
300 \text { B.C. }\end{array}$ & $\begin{array}{r}3.6 \\
\text { NA }\end{array}$ & 1900 & $\begin{array}{l}5910 \\
5910 \\
\end{array}$ & $\begin{array}{r}8.0 \\
\text { NA } \\
\end{array}$ & $\begin{array}{l}19.0 \\
19.0 \\
\end{array}$ & $\begin{array}{l}28.0 \\
22.0 \\
\end{array}$ & $\begin{array}{l}22 \\
100 \\
\end{array}$ & $3.0 / 3.0$ \\
\hline 3 & $\begin{array}{c}\text { Tisawewa } \\
300 \text { B.C. }\end{array}$ & $\begin{array}{l}\mathrm{NA} \\
2.0 \\
\end{array}$ & 2900 & $\begin{array}{l}11000 \\
11000 \\
\end{array}$ & $\begin{array}{r}18 \\
\text { NA }\end{array}$ & $\begin{array}{l}15.3 \\
13.5\end{array}$ & $\begin{array}{l}25.0 \\
19.5\end{array}$ & $\begin{array}{c}50 \\
100 \\
\end{array}$ & $2.8 / 3.0$ \\
\hline 4 & $\begin{array}{c}\text { Pavat Kulam } \\
300 \text { B.C. }\end{array}$ & $\begin{array}{l}\text { NA } \\
115 \\
\end{array}$ & 27000 & $\begin{array}{l}9700 \\
9700\end{array}$ & $\begin{array}{l}25.0 \\
\text { NA }\end{array}$ & $\begin{array}{l}18.0 \\
19.3 \\
\end{array}$ & $\begin{array}{l}28.0 \\
28.8 \\
\end{array}$ & $\begin{array}{r}250 \\
1060\end{array}$ & $3.0 / 2.5$ \\
\hline 5 & $\begin{array}{c}\text { Tissa wewa } \\
300 \text { B.C. }\end{array}$ & $\begin{array}{l}\text { NA } \\
15.7\end{array}$ & 2900 & $\begin{array}{l}4000 \\
4000 \\
\end{array}$ & $\begin{array}{c}20.00 \\
\text { NA }\end{array}$ & $\begin{array}{l}13.5 \\
15.5\end{array}$ & $\begin{array}{l}18.5 \\
18.0 \\
\end{array}$ & $\begin{array}{l}100 \\
200 \\
\end{array}$ & $5.0 / 4.5$ \\
\hline 6 & $\begin{array}{c}\text { Vavuni Kulam } \\
200 \text { B.C. }\end{array}$ & $\begin{array}{c}\text { NA } \\
88 \\
\end{array}$ & 35300 & $\begin{array}{l}13550 \\
13550 \\
\end{array}$ & $\begin{array}{l}12.0 \\
\text { NA }\end{array}$ & $\begin{array}{l}18.0 \\
24.0\end{array}$ & $\begin{array}{l}26.0 \\
32.0 \\
\end{array}$ & $\begin{array}{c}830 \\
2500 \\
\end{array}$ & $3.0 / 3.0$ \\
\hline 7 & Yoda wewa & $\begin{array}{r}18 \\
\text { NA }\end{array}$ & 7900 & $\begin{array}{l}3600 \\
3600 \\
\end{array}$ & $\begin{array}{l}15.0 \\
\text { NA }\end{array}$ & $\begin{array}{r}9.0 \\
8.25 \\
\end{array}$ & $\begin{array}{l}14.0 \\
12.7\end{array}$ & $\begin{array}{r}60.0 \\
163.0 \\
\end{array}$ & NA \\
\hline 8 & $\begin{array}{c}\text { Weerawila wewa } \\
100 \text { B.C. }\end{array}$ & $\begin{array}{c}\text { NA } \\
32 \\
\end{array}$ & 10000 & $\begin{array}{l}11400 \\
11400 \\
\end{array}$ & $\begin{array}{l}20.0 \\
\text { NA }\end{array}$ & $\begin{array}{l}12.5 \\
12.7 \\
\end{array}$ & $\begin{array}{l}17.5 \\
19.3 \\
\end{array}$ & $\begin{array}{l}\text { NA } \\
250 \\
\end{array}$ & $7.5 / 7.0$ \\
\hline 9 & $\begin{array}{c}\text { Batalagoda wewa } \\
100 \text { B.C. }\end{array}$ & $\begin{array}{c}\mathrm{NA} \\
5.0 \\
\end{array}$ & 4400 & $\begin{array}{l}4000 \\
4000 \\
\end{array}$ & $\begin{array}{l}5.0 \\
\text { NA }\end{array}$ & $\begin{array}{l}17.0 \\
12.7 \\
\end{array}$ & $\begin{array}{l}30.0 \\
19.3 \\
\end{array}$ & $\begin{array}{l}\text { NA } \\
250\end{array}$ & $3.0 / 2.5$ \\
\hline 10 & Nuwara wewa & $\begin{array}{l}\text { NA } \\
32.5\end{array}$ & 36000 & $\begin{array}{l}16000 \\
16000 \\
\end{array}$ & $\begin{array}{l}16.0 \\
\text { NA }\end{array}$ & $\begin{array}{c}17(31) \\
23 \\
\end{array}$ & $\begin{array}{l}37 \\
33 \\
\end{array}$ & $\begin{array}{l}136 \\
243 \\
\end{array}$ & $3.0 / 2.5$ \\
\hline 11 & $\begin{array}{c}\text { Nachchaduwa } \\
\text { wewa }\end{array}$ & $\begin{array}{l}\text { NA } \\
236\end{array}$ & 45100 & $\begin{array}{l}5500 \\
55 \mathrm{CO}\end{array}$ & $\begin{array}{l}20.0 \\
\text { NA }\end{array}$ & $\begin{array}{l}14.0 \\
15.0\end{array}$ & $\begin{array}{l}36.0 \\
25.0\end{array}$ & $\begin{array}{l}167 \\
466\end{array}$ & $2.5 / 2.5$ \\
\hline
\end{tabular}

NA - Not Available, Top row shows parameters for ancient tanks and bottom row shows parameters for restored tanks. 


\section{Results}

The calculation of parameters for $\mathrm{H}=\mathrm{BTL}-\mathrm{FSL}$, H - BTL - FSL - 2, H = BTL - FSL - 4 are shown in Tables 2(a), 2(b) and 2(c) respectively.

Table 2(a) Spill analysis using Arumugam (1957), H=BTL-FSL, Ancient Tanks.

\begin{tabular}{|l|c|c|c|c|c|c|c|c|}
\hline & $\begin{array}{c}\text { Catchment } \\
\text { Area - M } \\
- \text { sq miles }\end{array}$ & $\begin{array}{c}\text { Flood } \\
\text { inflow } \\
\mathbf{Q}=\mathrm{CM}^{3 / 4}\end{array}$ & $\begin{array}{c}\text { Spill } \\
\text { Length - }-(\mathbf{f t})\end{array}$ & $\begin{array}{c}\mathrm{H} \\
(\mathrm{ft})\end{array}$ & $\begin{array}{c}\text { Spill= } \\
3.3 \mathbf{L H}^{3 / 2} \\
(\mathrm{acre} \mathbf{f t})\end{array}$ & $\begin{array}{c}\text { Area - A - } \\
\text { (acres) }\end{array}$ & $\begin{array}{c}\text { Detent- } \\
\text { ion - AH - } \\
\text { (acre ft) }\end{array}$ & $\begin{array}{c}\text { Detention } \\
+ \text { Spill } \\
(\text { acre ft) }\end{array}$ \\
\hline Nachchaduwa wewa & 236 & 48170 & 167 & 22 & 56868 & 2015 & 44330 & 101198 \\
\hline Pavat Kulum & 115 & 28094 & 250 & 10 & 26089 & 2029 & 20290 & 46379 \\
\hline Vavuni Kulum & 88 & 22985 & 530 & 8 & 39575 & 1975 & 15800 & 55375 \\
\hline Nuwara wewa & 32.5 & 10889 & 136 & 6 & 6596 & 3180 & 19080 & 25676 \\
\hline Yoda wewa & 18 & 6991 & 60 & 5 & 2214 & 1230 & 6150 & 8364 \\
\hline Tissa wewa & 15.7 & 6310 & 100 & 5 & 3690 & 652 & 3260 & 6950 \\
\hline Basawakkulama Tank & 3.6 & 2091 & 22 & 9 & 1960 & 330 & 2970 & 4930 \\
\hline Tisa wewa & 2 & 1345 & 50 & 9.7 & 4985 & 396 & 3841 & 8826 \\
\hline
\end{tabular}

Table 2(b) Spill analysis using Arumugam (1957), H=BTL-FSL-2, Ancient Tanks.

\begin{tabular}{|c|c|c|c|c|c|c|c|c|}
\hline & $\begin{array}{c}\text { Catchment } \\
\text { Area - M } \\
\text { - sq miles }\end{array}$ & $\begin{array}{l}\text { Flood } \\
\text { inflow } \\
\mathrm{Q}=\mathrm{CM}^{3 / 4}\end{array}$ & $\begin{array}{c}\text { Spill } \\
\text { Length - } \\
\text { L - (fft) }\end{array}$ & $\begin{array}{l}\mathrm{H} \\
(\mathrm{ft})\end{array}$ & $\begin{array}{l}\text { Spill = } \\
3.3 \mathrm{LH}^{3 / 2} \\
(\text { acre } \mathbf{f t})\end{array}$ & $\begin{array}{c}\text { Area - A - } \\
\quad(\text { acres })\end{array}$ & $\begin{array}{c}\text { Detent- } \\
\text { ion - AH - } \\
\text { (acreft) }\end{array}$ & $\begin{array}{c}\text { Detention } \\
+ \text { Spill } \\
(\text { acre } \mathrm{ft})\end{array}$ \\
\hline Nachrhaduwawewa & 236 & 48170 & 167 & 20 & 49292 & 2015 & 40300 & 89592 \\
\hline Pavat Kulum & 115 & 28094 & 250 & 8 & 18668 & 2029 & 16232 & 34900 \\
\hline Vavuni Kulum & 88 & 22985 & 530 & 6 & 25705 & 1975 & 11850 & 37555 \\
\hline Nuwarn wewa & 32.5 & 10889 & 136 & 4 & 3590 & 3180 & 12720 & 16310 \\
\hline Yoda wcwa & 18 & 6991 & 60 & 3 & 1029 & 1230 & 3690 & 4719 \\
\hline Tissa wcwa & 15.7 & 6310 & 100 & 3 & 1715 & 652 & 1956 & 3671 \\
\hline Basawakkulama Tank & 3.6 & 2091 & 22 & 7 & 1345 & 330 & 2310 & 3655 \\
\hline Tisa wewa & 2 & 1345 & 50 & 7.7 & 3525 & 396 & 3049 & 6575 \\
\hline
\end{tabular}


Table 2(c) Spill analysis using Arumugam (1957), H=BTL-FSL-4, Ancient Tanks.

\begin{tabular}{|c|c|c|c|c|c|c|c|c|}
\hline & $\begin{array}{c}\text { Catchment } \\
\text { Area - M } \\
\text { - sq miles }\end{array}$ & $\begin{array}{c}\text { Flood } \\
\text { inflow } \\
\mathrm{Q}=\mathrm{CM}^{3 / 4}\end{array}$ & $\begin{array}{c}\text { Spill } \\
\text { Length - } \\
\text { L - (ft) }\end{array}$ & $\begin{array}{l}\mathrm{H} \\
(\mathrm{ft})\end{array}$ & $\begin{array}{l}\text { Spill = } \\
3.3 \mathrm{LH}^{3 / 2} \\
(\text { acre } \mathrm{ft})\end{array}$ & $\begin{array}{c}\text { Area - A - } \\
\quad \text { (acres) }\end{array}$ & $\begin{array}{c}\text { Detent- } \\
\text { ion - AH - } \\
\text { (acre } \mathrm{ft})\end{array}$ & $\begin{array}{c}\text { Detention } \\
+ \text { Spill } \\
\text { (acre } \mathbf{f t})\end{array}$ \\
\hline Nachchaduwa wewa & 236 & 48170 & 167 & 18 & 42086 & 2015 & 36270 & 78356 \\
\hline Pavat Kulum & 115 & 28094 & 250 & 6 & 12125 & 2029 & 12174 & 24299 \\
\hline Vavuni Kulum & 88 & 22985 & 530 & 4 & 13992 & 1975 & 7900 & 21892 \\
\hline Nuwara wewa & 32.5 & 10889 & 136 & 2 & 1269 & 3180 & 6360 & 7629 \\
\hline Yoda wewa & 18 & 6991 & 60 & 1 & 198 & 1230 & 1230 & 1428 \\
\hline Tissa wewa & 15.7 & 6310 & 100 & 1 & 330 & 652 & 652 & 982 \\
\hline Basawakkulama Tank & 3.6 & 2091 & 22 & 5 & 812 & 330 & 1650 & 2462 \\
\hline Tisa wewa & 2 & 1345 & 50 & 5.7 & 2245 & 396 & 2257 & 4503 \\
\hline
\end{tabular}

Table 2(d) Summary of results for ancient tanks

\begin{tabular}{|l|c|c|c|c|}
\hline & \multirow{2}{*}{$\begin{array}{c}\text { Flood inflow } \\
\text { Q=CM }\end{array}$} & \multicolumn{3}{|c|}{ Detention + spill (Acre ft) } \\
\cline { 3 - 5 } & 48170 & BTL-FSL & BTL-FSL-2 & BTL-FSL-4 \\
\hline Nachchaduwa wewa & 28094 & 101198 & 89592 & 78356 \\
\hline Pavat Kulum & 22985 & 46379 & 34900 & 24299 \\
\hline Vavuni Kulum & 10889 & 25676 & 37555 & 21892 \\
\hline Nuwara wewa & 6991 & 8364 & 16310 & 7629 \\
\hline Yoda wewa & 6310 & 6950 & 4719 & 1428 \\
\hline Tissa wewa & 2091 & 4930 & 3671 & 982 \\
\hline Basawakkulama Tank & 1345 & 8826 & 3655 & 2462 \\
\hline Tisa wewa & & 6575 & 4503 \\
\hline
\end{tabular}

The calculation of parameters for restored tanks for $\mathrm{H}=\mathrm{BTL}$ - FSL, $\mathrm{H}=\mathrm{BTL}-\mathrm{FSL}-2, \mathrm{H}=\mathrm{BTL}$ FSL -4 are shown in Tables $3(\mathrm{a}), 3(\mathrm{~b})$ and $3(\mathrm{c})$ respectively.

Table 3(a) Spill analysis using Arumugam (1957), H=BTL-FSL, Ancient Tanks after restoration

\begin{tabular}{|c|c|c|c|c|c|c|c|c|}
\hline & $\begin{array}{c}\text { Catchment } \\
\text { Area - M } \\
\text { - sq miles }\end{array}$ & $\begin{array}{c}\text { Flood } \\
\text { inflow } \\
\mathrm{Q}=\mathrm{CM}^{3 / 4}\end{array}$ & $\begin{array}{c}\text { Spill } \\
\text { Length - } \\
\mathrm{L}-(\mathrm{ft})\end{array}$ & $\begin{array}{c}\mathrm{H} \\
(\mathrm{ft})\end{array}$ & $\begin{array}{l}\text { Spill = } \\
3.3 \mathrm{LH}^{3 / 2} \\
(\text { acre } \mathbf{f t})\end{array}$ & $\begin{array}{c}\text { Area - A - } \\
\text { (acres) }\end{array}$ & $\begin{array}{c}\text { Detent- } \\
\text { ion - AH - } \\
\text { (acre ft) }\end{array}$ & $\begin{array}{c}\text { Detention } \\
+ \text { Spill } \\
(\text { acre } \mathrm{ft})\end{array}$ \\
\hline Nachchaduwa wewa & 236 & 48170 & 466 & 10 & 48630 & 4400 & 44000 & 92630 \\
\hline Pavat Kulum & 115 & 28094 & 1060 & 9.5 & 102425 & 3000 & 28500 & 130925 \\
\hline Vavuni Kulum & 88 & 22985 & 2500 & 8 & 186676 & 3150 & 25200 & 211876 \\
\hline Nuwara wewa & 32.5 & 10889 & 243 & 10 & 25358 & 3000 & 30000 & 55358 \\
\hline Yoda wewa & 18 & 6991 & 163 & 4.45 & 5049 & 1200 & 5340 & 10389 \\
\hline Tissa wewa & 15.7 & 6310 & 200 & 2.5 & 2609 & 575 & 1438 & 4046 \\
\hline Basawakkulama Tank & 3.6 & 2091 & 100 & 5.5 & 4257 & 265 & 1458 & 5714 \\
\hline Tisa wewa & 2 & 1345 & 100 & 6 & 4850 & 450 & 2700 & 7550 \\
\hline
\end{tabular}


Table 3(b) Spill analysis using Arumugam (1957), H=BTL-FSL-2, Ancient Tanks after restoration

\begin{tabular}{|l|c|c|c|c|c|c|c|c|}
\hline & $\begin{array}{c}\text { Catchment } \\
\text { Area - M } \\
- \text { sq miles }\end{array}$ & $\begin{array}{c}\text { Flood } \\
\text { inflow } \\
\mathbf{Q}=\mathrm{CM}^{3 / 4}\end{array}$ & $\begin{array}{c}\text { Spill } \\
\text { Length - } \\
\text { L (ft) }\end{array}$ & $\begin{array}{c}\mathrm{H} \\
(\mathrm{ft})\end{array}$ & $\begin{array}{c}\text { Spill= } \\
3.3 \mathbf{L H}^{3 / 2} \\
(\text { acre ft) }\end{array}$ & $\begin{array}{c}\text { Area - A - } \\
(\text { acres })\end{array}$ & $\begin{array}{c}\text { Detent- } \\
\text { ion - AH - } \\
\text { (acreft) }\end{array}$ & $\begin{array}{c}\text { Detention } \\
+ \text { Spill } \\
(\text { acre ft) }\end{array}$ \\
\hline Nachchaduwawewa & 236 & 48170 & 466 & 8 & 34796 & 4400 & 35200 & 69996 \\
\hline Pavat Kulum & 115 & 28094 & 1060 & 7.5 & 71848 & 3000 & 22500 & 94348 \\
\hline Vavuni Kulum & 88 & 22985 & 2500 & 6 & 121250 & 3150 & 18900 & 140150 \\
\hline Nuwara wewa & 32.5 & 10889 & 243 & 8 & 18145 & 3000 & 24000 & 42145 \\
\hline Yoda wcwa & 18 & 6991 & 163 & 2.45 & 2063 & 1200 & 2940 & 5003 \\
\hline Tissa wewa & 15.7 & 6310 & 200 & 0.5 & 233 & 575 & 288 & 521 \\
\hline Basawakkulama Tank & 3.6 & 2091 & 100 & 3.5 & 2161 & 265 & 928 & 3088 \\
\hline Tisa wewa & 2 & 1345 & 100 & 4 & 2640 & 450 & 1800 & 4440 \\
\hline
\end{tabular}

Table 3(c) Spill analysis using Arumugam (1957), H=BTL-FSL-4, Ancient Tanks after restoration

\begin{tabular}{|l|c|c|c|c|c|c|c|c|}
\hline & $\begin{array}{c}\text { Catchment } \\
\text { Area - M } \\
- \text { sq miles }\end{array}$ & $\begin{array}{c}\text { Flood } \\
\text { inflow } \\
\mathbf{Q}=\mathbf{C M}^{3 / 4}\end{array}$ & $\begin{array}{c}\text { Spill } \\
\text { Length - }-(\mathbf{f t})\end{array}$ & $\begin{array}{c}\mathrm{H} \\
(\mathrm{ft})\end{array}$ & $\begin{array}{c}\text { Spill= } \\
3.3 \mathbf{L H}^{3 / 2} \\
(\mathrm{acre} \mathbf{f t})\end{array}$ & $\begin{array}{c}\text { Area - A - } \\
(\text { acres })\end{array}$ & $\begin{array}{c}\text { Detent- } \\
\text { ion - AH - } \\
(\text { acre ft) }\end{array}$ & $\begin{array}{c}\text { Detention } \\
+ \text { Spill } \\
(\mathrm{acre} \mathbf{f t})\end{array}$ \\
\hline Nachchaduwa wewa & 236 & 48170 & 466 & 6 & 22601 & 4400 & 26400 & 49001 \\
\hline Pavat Kulum & 115 & 28094 & 1060 & 5.5 & 45119 & 3000 & 16500 & 61619 \\
\hline Vavuni Kulum & 88 & 22985 & 2500 & 4 & 66000 & 3150 & 12600 & 78600 \\
\hline Nuwara wewa & 32.5 & 10889 & 243 & 6 & 11785 & 3000 & 18000 & 29785 \\
\hline Yoda wewa & 18 & 6991 & 163 & 0.45 & 162 & 1200 & 540 & 702 \\
\hline Tissa wewa & 15.7 & 6310 & 200 & 0 & 0 & 575 & 0 & 0 \\
\hline Basawakkulama Tank & 3.6 & 2091 & 100 & 1.5 & 606 & 265 & 398 & 1004 \\
\hline Tisa wewa & 2 & 1345 & 100 & 2 & 933 & 450 & 900 & 1833 \\
\hline
\end{tabular}


The plots of flood inflow vs (detention + Spill)

for all 6 cases in Tables 2(a), 2(b), 2(c), 3(a), 3(b)

and $3(\mathrm{c})$ are as shown in Fig. 3.

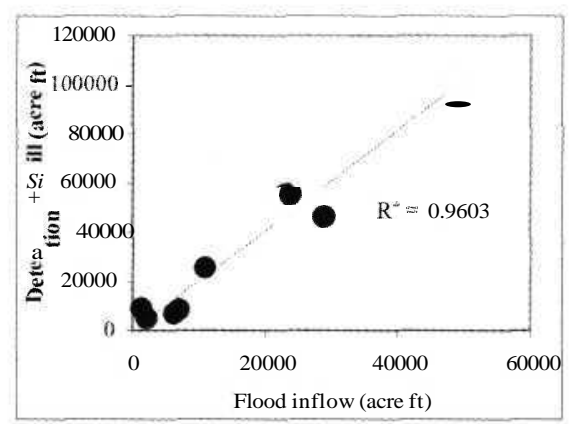

(a)

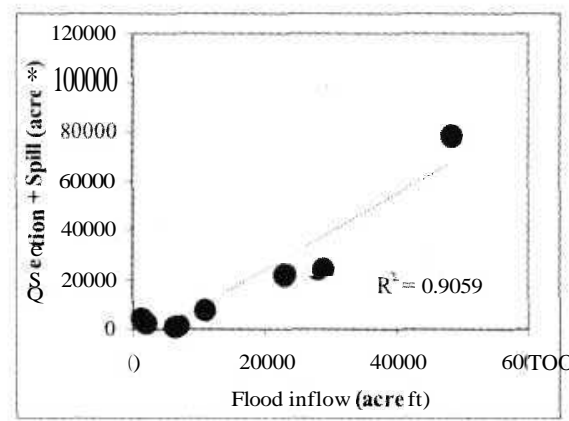

(c)

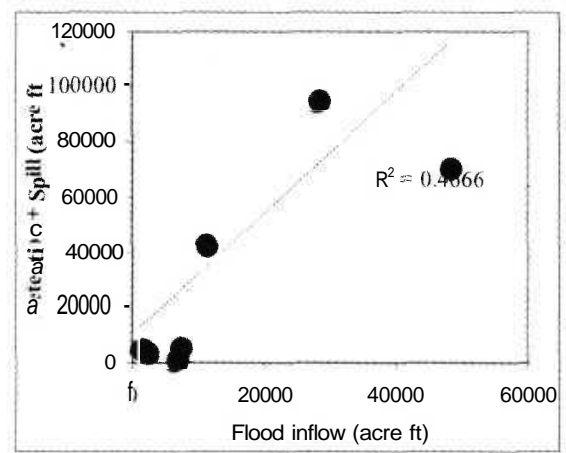

(e)

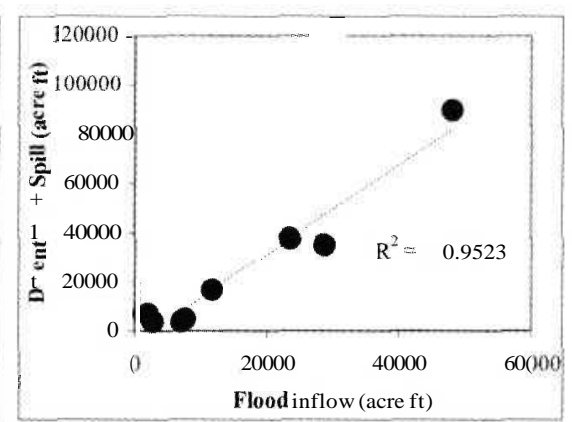

(b)

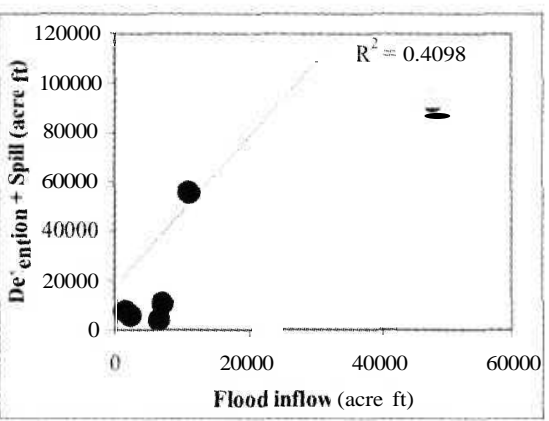

(d)

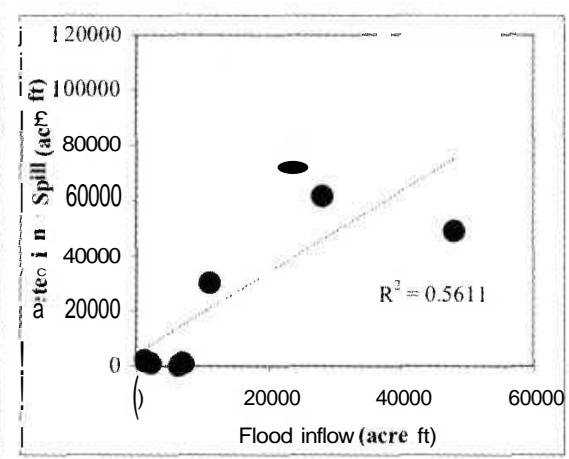

(f)

Fig. 3 - Plots of flood inflow vs (detention + spill) for ancient tanks $(a, b$ and $\mathrm{c})$ and for ancient tanks after restoration $(\mathrm{d}$, e and $\mathrm{f})$ 


\section{Concluding Discussion}

As seen from the graphs and also from Table 4 below (which gives a summary of correlation coefficientsfor all 6 cases the correlation between the flood inflow and (detention + Spill) is very good $(>0.90)$ for ancient tanks. Even for restored tanks the coefficients are greater than 0.4.

Table 4 - Correlation co-efficient (when $\mathrm{C}=\mathbf{8 0 0}$ )

\begin{tabular}{|l|c|c|}
\hline \multicolumn{1}{|c|}{ Tank } & $\begin{array}{c}\text { Head over spill } \\
\text { (ft) }\end{array}$ & $\begin{array}{c}\text { Correlation } \\
\text { co-efficient } \\
\left(\mathrm{R}^{2}\right)\end{array}$ \\
\hline Ancient & H=BTL-FSL & $\mathbf{0 . 9 6}$ \\
tanks & H= BTL-FSL - 2ft & $\mathbf{0 . 9 5}$ \\
& H=BTL-FSL -4ft & $\mathbf{0 . 9 1}$ \\
\hline Ancient & H=BTL-FSL & $\mathbf{0 . 4 1}$ \\
Links after & H= BTL-FSL -2ft & $\mathbf{0 . 4 7}$ \\
restoration & H=BTL-FSL - 4ft & $\mathbf{0 . 5 6}$ \\
\hline
\end{tabular}

The correlation coefficient is considered strong if for a sample size of 5 the minimum $R^{2}=0.77$ and for a sample size of 10 the minimum $R^{2}$ is 0.40 (Pardoe, 1962). From the above figures, we see that both ancient tanks and present tanks show, correlation. The stronger correlation is with the ancient tanks. The difference between the two cases is that in the case of the present tanks, the design was done by present day engineers using advanced hydrological studies using unit graphs and flood routing through spills.

A summary of the results of flood inflow (for $\mathrm{C}=$ 800) and the (detention + spill) is given in Table 2(d). This Table shows that 5 of the ancient tanks lie within the range BTL-FSL and BTL - FSL - 4ft. The 3 tanks Nachchaduwa, Basawakkulama and Tisawewahave high BTL- FSL. Freeboards higher than $4 \mathrm{ft}$ may have been used in their design. This is probably why they lie outside the range $\mathrm{BTL}$ FSL and BTL - FSL - 4ft.

In view of the very strong correlation for ancient tanks and with 5 out of 8 tanks falling within the range of $\mathrm{H}=\mathrm{BTL}$ - FSL and $\mathrm{H}=\mathrm{BTL}$ - FSL - 4ft, can we say that the ancient builders very likely used the simple Arumugam method for designing their tanks? The spill discharge and detention formula musthave been known to them as they are simple in concept. However, the ancient builders would not have had information about the catchment areas. They would have estimated the flood inflow by gauging of the river during flood.
Mr DGL Ranatunga states that "the Egyptians took levels on the Nile as far back as 3000 BC. They subsequently developed techniques for flood warning, hydraulics etc. Hence it may not be farfetched to imagine this to be a clue to the origin of irrigation in Sri Lanka.

In the gauging of flood inflow, during floods, the high flood level has to be estimated by observation of twigs and other telltale marks left by the floods. The area of flow has to be measured. The velocity of flow has to be obtained by the distance covered by floods over a period of time or a velocity of $12 \mathrm{ft} / \mathrm{s}$ may have been used. Area velocity will give the flood inflow very approximately.

As regards Dickens $\mathrm{C}=800$ used, we give a Table (Table 5) - for Mahakanadarawa floods. Mahakanadarawa tank is near Mihintale in the dry zone. It has a catchment are of 133 sq miles.

Table 5 - Dickens C for Mahakanadarawa Tank

\begin{tabular}{|c|c|c|}
\hline $\begin{array}{c}\text { Flood } \\
\text { magnitude }\end{array}$ & Flood inflow & Dickens C \\
\hline 100 year & 60,000 & 1500 \\
\hline 50 year & 45,000 & 1200 \\
\hline 25 year & 31,000 & 800 \\
\hline
\end{tabular}

Thus $\mathrm{C}=800$ corresponds approximately to a flood of 25 years only.

So the ancient builders around $300 \mathrm{BC}$ may have used the Arumugam method for the earliest irrigation tanks. The other possibility is that during the restoration of the many tanks by Parakrama Bahu the great 1153-1186 AD this method may have been used. The spill lengths and bund heights were adjusted to suit. The flood inflows would have been obtained by studying the spill discharge heights during the operation of the tanks over 1400 years, except when breached.

This analysis was carried out for the 8 tanks shown in Fig. 1 as the required data could be obtained only for these 8 tanks. However, it will be interesting to see if the hypothesis holds true for other ancient tanks as well. 


\section{References}

1. Arumugam S (1957), "Development of Village Irrigation Works" Transactions IESL 1957.

2. Arumugam, S (1969). "Water Resources of Ceylon". Water Resources Board, Sri Lanka.

3. Dharmasena GT (1988). "Some Aspects of Ancient Irrigation Works in Sri Lanka", Engineer, Sep/Dec 1998.

4. Pardoe Kevin (1962). "Quantitative Methods", Butterworth-Heinemann, New-York.

5. Parker H (1909) Ancient Ceylon, Reprint Asian Educational Services, New Delhi., 1992.

6. Ranatunga DGL (1973). "Contributions of the ancient civilizations of Sri Lanka to the Historical Development of Hydrology", Engineer, December 1973. 